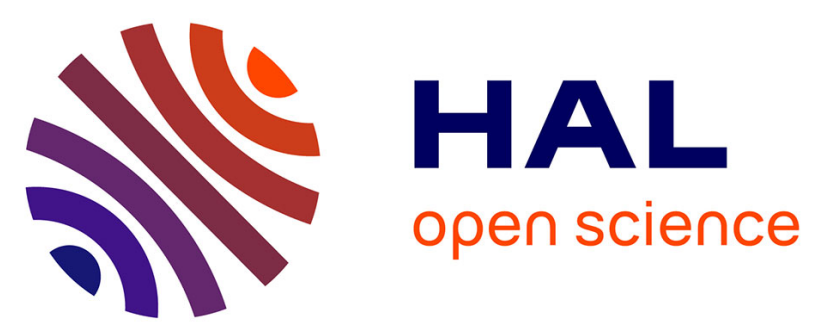

\title{
Global Throughput Maximization of a Hybrid Unicast-Broadcast Network for Linear Services
}

Pape Abdoulaye Fam, Matthieu Crussière, Jean-François Hélard, Pierre Brétillon, Stéphane Paquelet

\section{- To cite this version:}

Pape Abdoulaye Fam, Matthieu Crussière, Jean-François Hélard, Pierre Brétillon, Stéphane Paquelet. Global Throughput Maximization of a Hybrid Unicast-Broadcast Network for Linear Services. 12th International Symposium on Wireless Communication Systems 2015, Aug 2015, Bruxelles, Belgium. 10.1109/ISWCS.2015.7454316 . hal-01279713

\section{HAL Id: hal-01279713 \\ https://hal.science/hal-01279713}

Submitted on 26 Feb 2016

HAL is a multi-disciplinary open access archive for the deposit and dissemination of scientific research documents, whether they are published or not. The documents may come from teaching and research institutions in France or abroad, or from public or private research centers.
L'archive ouverte pluridisciplinaire HAL, est destinée au dépôt et à la diffusion de documents scientifiques de niveau recherche, publiés ou non, émanant des établissements d'enseignement et de recherche français ou étrangers, des laboratoires publics ou privés. 


\title{
Global Throughput Maximization of a Hybrid Unicast-Broadcast Network for Linear Services
}

\author{
Pape-Abdoulaye Fam ${ }^{* \ddagger}$, Matthieu Crussière ${ }^{* \dagger}$, Jean-François Hélard ${ }^{\dagger}$, Pierre Brétillon ${ }^{\ddagger}$ and Stéphane Paquelet ${ }^{*}$ \\ *b<>com, 1219 avenue des Champs Blancs, 35510 Cesson-Sévigné, France \\ ${ }^{\dagger}$ INSA, IETR, UMR 6164, F-35708 RENNES, France \\ †TDF, Centre d'affaires CESCOM, 4, rue Marconi - BP 25180, 57075 METZ Cedex 03, France \\ Email: pape-abdoulaye.fam@b-com.com
}

\begin{abstract}
An exponential growth of the mobile data traffic is expected in future networks. The reason of this growth is related to the increasing popularity of linear services such as mobile TV, live and sports events, which may lead to the delivery of the same contents to a large audience. Recent studies have shown that network cooperation is a promising candidate to deal with such an issue. This paper investigates, from a planning perspective, the optimization of a hybrid unicast/broadcast network for linear services. We introduce an analytical approach with the aim of stating an optimization problem on the hybrid network configuration, namely in terms of the sharing between the unicast and broadcast modes to deliver a particular service. We show by simulation the existence of an optimal operation point which leads to the maximization of the overall throughput of the hybrid network under the constraint of a minimum service success rate.
\end{abstract}

\section{INTRODUCTION}

According to the data traffic forecast by Cisco [1], an exponential growth of the data traffic in mobile broadband networks is expected in a near future. As a matter of fact, due to the increase of large screen devices, a significant amount of this traffic is driven by video services. Depending on their popularity, these services could be considered as linear, meaning that the same contents may be delivered to a large audience at the same time. In mobile broadband networks, linear services are usually associated to "on-demand" usage and delivered over unicast connections, i.e. as many times as the number of users. This strategy is likely to lead to critical situations, e.g. network congestion, while a broadcast bearer would be more suitable to accommodate such kind of traffic.

In this context, a mixed unicast and broadcast approach is under study within the 3GPP Long Term Evolution consortium through the definition of the evolved Multimedia Broadcast Muliticast (eMBMS) transmission mode [2]. Another promising strategy is to enable cooperation between mobile broadband networks and terrestrial TV broadcast networks. This latter case refers to the definition of a hybrid network taking advantage of not only various types of technologies but also various types of network infrastructures. Recent studies in the literature have for instance addressed the cooperation between 3GPP and DVB networks. As a example, a common physical layer with a flexible waveform is specified in [3] to provide a unified system suitable for mobile broadband and TV broadcast networks. Complementary to this, [4] proposes a cooperation scenario in which LTE broadcast services are offloaded to a DVB network using the concept of LTE carrier aggregation and DVB-T2 future extension frame. In these studies, as in many others dealing with network cooperation, investigations have been mainly focused on network selection decision and often assume a common coverage area for the mobile broadband and broadcast networks. However, authors in [5] investigate, from a planning perspective, the case in which the networks have different coverage areas. In this case the LTE-eMBMS mode is used to extend the coverage of services initially delivered through the broadcast network. It is then shown that the service coverage extension concept is the optimal cooperation strategy in terms of global capacity enhancement, power consumption reduction [6] and quality of service improvement [7]. However, these latter works consider broadcast transmissions for both the broadcast and the mobile broadband networks.

This paper proposes to study further the service coverage extension scenario by considering an hybrid unicast/broadcast network in which a unicast transmission mode is associated to the mobile broadband network and a broadcast transmission mode to the terrestrial TV broadcast network. This paper aims at showing the existence of an optimal operation mode for which the overall capacity of the hybrid network is optimized. In that perspective we state an optimization problem that takes into account the characteristics of the hybrid network and the user distribution. A major contribution of this paper is to provide the optimal terrestrial TV broadcast planning parameters for a given quality of service requirement.

The rest of this paper is organized as follows. In section II, we introduce the hybrid model and define several metrics used in simulations. Then, section III introduces the analytical approach to state the optimization problem from a planning point of view. Section IV presents the simulation results. Finally section $\mathrm{V}$ concludes the paper and provides insight on future works.

\section{HYBRID NETWORK MODEL}

We consider a transmission of linear services to a set of devices in a given region of interest (ROI) as depicted in Fig. 1. A minimum throughput $R_{s}$ is required for a user to receive the service. A broadcast network and a mobile broadband network are considered, both of which are based on Orthogonal Frequency Division Multiplexing (OFDM). Unicast transmission mode only is assumed for the mobile broadband network which is referred to as the unicast network for the rest of the paper. It is composed of $N_{L P L T}$ Low Power Low Tower (LPLT) stations. On the other hand, the broadcast network which is responsible for the broadcast transmission mode, is composed of one single High Power High Tower (HPHT) station with a coverage radius $R$. The proposed services are assumed to be requested by $M$ users in the region of interest. A user is attached to a LPLT or HPHT station depending on 


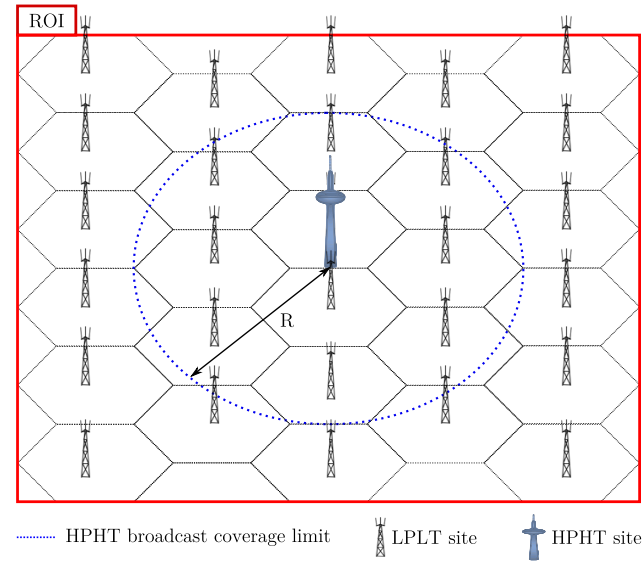

Fig. 1. Hybrid network model

the signal to noise plus interference ratio (SINR) that occurs on each link between that user and the surrounding stations. More precisely, users located within the broadcast network coverage area defined by its radius $R$ are attached to the HPHT transmitter. The other users are attached to the LPLT station of the unicast network that gives the highest SINR.

\section{A. SINR computations at a given position in the ROI}

In OFDM systems, the SINR of a user $m$ connected to a station $n$ is calculated for each subcarrier $k$ as

$$
\gamma_{k}(m, n)=\frac{P_{n, k} L\left(d_{m, n}\right)}{N_{0} \Delta_{f}+I_{k}}
$$

where $P_{n, k}$ is the power associated to station $n$ and subcarrier $k, d_{m, n}$ is the distance between user $m$ and station $n$, $L\left(d_{m, n}\right)=\left(\lambda /\left(4 \pi d_{m, n}\right)\right)^{\alpha}$ is the path loss between station $n$ and user $m$ with $\alpha$ the path loss exponent, $N_{0}$ is the background noise level, $\Delta_{f}$ is the subcarrier spacing and $I_{k}$ the average inter-cell interference power on the $k^{t h}$ subcarrier.

Inter-cell interference is taken into account for the unicast network as a frequency reuse factor of 1 is used for LPLT cells, i.e. all cells use the same frequency resources. This inter-cell interference issue is not considered for the broadcast network as a single HPHT station is used. Therefore the SINR per subcarrier for a user connected to the broadcast network is replaced by the signal to noise ratio (SNR), which is obtained from (1) by omitting the interference term $I_{k}$,

$$
\operatorname{SNR}_{k}(m, n)=\frac{P_{n, k} L\left(d_{m, n}\right)}{N_{0} \Delta_{f}}
$$

Note that fast fading and shadowing effects are ignored in (1) and (2). Inter-system interference is also ignored because the broadcast and the unicast networks operate in different frequency bands.

In multicarrier systems, a convenient approach is to use the effective SINR mapping concept based on the following equivalent SINR expression [8]:

$$
\gamma_{e f f}(m, n)=I^{-1}\left[\frac{1}{K_{m, n}} \sum_{k \in \mathcal{K}_{m, n}} I\left(\gamma_{k}(m, n)\right)\right]
$$

where $I($.$) corresponds to an "information measure" function$ and $I^{-1}($.$) is its inverse, \mathcal{K}_{m, n}$ and $K_{m, n}$ are respectively the set and the number of subcarriers allocated to a user $m$ at station $n$. The equivalent SINR is used for network planning, resource allocation and throughput calculations.

\section{B. Throughput computations of a user}

When user $m$ is connected to a unicast station $n$, its throughput is obtained as

$$
r_{m, n}=C\left(\gamma_{e f f}(m, n), B_{m, n}\right)
$$

where $B_{m, n}$ is the bandwidth (i.e. the number of subcarriers) allocated to user $m$ by station $n$ and $C($.$) is a SINR$ to throughput mapping function. For instance, the Shannon channel capacity formula is widely used as a mapping function:

$$
C\left(\gamma_{e f f}(m, n), B_{m, n}\right)=B_{m, n} \log _{2}\left(1+\gamma_{e f f}(m, n)\right)
$$

Eq. (5) will be used to state the optimization problem in the next section. In practical systems, e.g. LTE, the effective SINR of each user is mapped to a channel quality indicator (CQI) using link level performance curves. A modulation and coding scheme (MCS) is chosen based on the reported CQI and the quality of service (QoS) requirements. Then the user throughput $r_{m, n}$ is calculated from the chosen MCS and the bandwidth allocated to the user by the station. This approach will be used in our simulations to closely reflect the behavior of a real system.

As far as the broadcast network is considered, all available subcarriers are allocated to all users. Link adaptation is not used for broadcast transmissions. Thus, all users will have the same throughput simply depending on the transmission power and the coverage area. In general, a broadcast network is planned such that a user located at the edge of the coverage area can receive the services. The throughput of a user connected to the broadcast network is then given by

$$
r_{B}=C\left(S N R_{\min }, B_{B}\right)
$$

where $S N R_{\text {min }}$ is the minimum effective SNR of a user located at the edge of the broadcast coverage area and $B_{B}$ is the total bandwidth allocated for broadcast transmission. Note that $S N R_{\min }$ is related to the coverage radius $R$ by

$$
R=\frac{\lambda}{4 \pi} \exp \left[-\frac{1}{\alpha} \log \left(\frac{N_{0} \Delta_{f}}{P_{n}} S N R_{\text {min }}\right)\right]
$$

where $P_{n}$ is the total transmission power at the broadcast station. (7) is obtained from (2) and using pathloss $L(R)$. In the sequel, $P_{n}$ will be assumed as fixed, so that $R$ will only depend on $S N R_{m i n}$.

\section{Metrics}

To evaluate the performance of the hybrid network, let us define the service success rate and service capacity.

Service success rate: The throughput of a user in the ROI is calculated using (4) if the user is connected to a unicast station or (6) if the user is connected to the broadcast station. Let $\mathcal{M}$ be the set of users and $\mathcal{M}^{(s)}=\mathcal{M}_{U}^{(s)} \cup \mathcal{M}_{B}^{(s)}$ be the subset of users that have a throughput greater than the required service throughput $R_{s}$, with $\mathcal{M}_{U}^{(s)}$ and $\mathcal{M}_{B}^{(s)}$ the subsets of users that receive the service through unicast and broadcast networks, respectively. The service success rate metric is then defined as

$$
\eta^{(s)}=\frac{M^{(s)}}{M}=\frac{M_{U}^{(s)}+M_{B}^{(s)}}{M}
$$

where, $M=\operatorname{Card}\{\mathcal{M}\}, M^{(s)}=\operatorname{Card}\left\{\mathcal{M}^{(s)}\right\}, M_{U}^{(s)}=$ $\operatorname{Card}\left\{\mathcal{M}_{U}^{(s)}\right\}$ and $M_{B}^{(s)}=\operatorname{Card}\left\{\mathcal{M}_{B}^{(s)}\right\}$. 
Service capacity: The service capacity is defined as the total throughput achieved by users that have access to the services, which writes

$$
C_{\text {service }}=M_{B}^{(s)} \times r_{B}+\sum_{m \in \mathcal{M}_{U}^{(s)}} r_{m, n}
$$

\section{THROUGHPUT MAXIMIZATION PROBLEM}

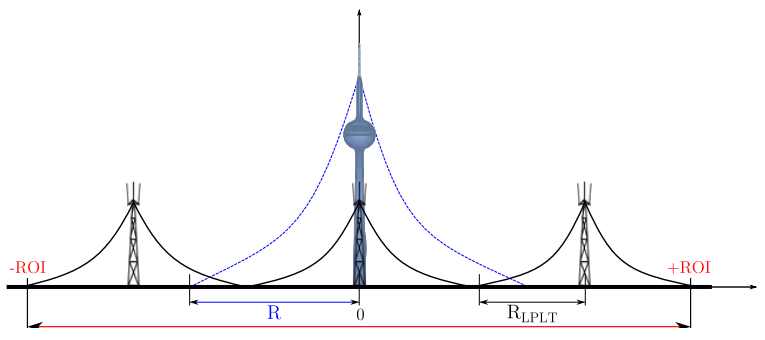

Fig. 2. One dimensionnal network model.

An analytical approach is introduced in this section to state the maximization problem which will then be solved through simulations.

Given $\mathcal{M}$ the set of users that are interested in a particular service, the problem is to find the subsets of users $\mathcal{M}_{U}^{(s)}$ and $\mathcal{M}_{B}^{(s)}$ that maximize the service capacity of the hybrid network such that a minimum service success rate is guaranteed, i.e. $\eta^{(s)} \geq \eta_{\min }^{(s)}$. Since the users located in the broadcast coverage area are served by the broadcast station while the rest of users are served by the unicast stations, one may easily understand that the numbers of users in the subsets $\mathcal{M}_{U}^{(s)}$ and $\mathcal{M}_{B}^{(s)}$ are directly related to the broadcast coverage area, i.e. $S N R_{\text {min }}$. Thus, $\mathcal{M}_{U}^{(s)}$ and $\mathcal{M}_{B}^{(s)}$ are determined by adjusting the coverage area of the broadcast network. Without loss of generality let us focus on the one dimensional model of the hybrid network as shown in Fig. 2. The capacity of the hybrid network is given by

$$
C_{h y b r i d}=M \times \mathrm{E}\left[C_{m}\right]
$$

where $\mathrm{E}[$.$] stands for expectation and,$

$$
C_{m}= \begin{cases}C\left(S N R_{\text {min }}, B_{B}\right) & \text { if } m \in \mathcal{M}_{B}, \\ C\left(\gamma_{e f f}(m, n), B_{m, n}\right) & \text { if } m \in \mathcal{M}_{U}(n) .\end{cases}
$$

with $\mathcal{M}_{B}$ and $\mathcal{M}_{U}(n)$ the sets of users connected to the broadcast station and to the $n$th station of the unicast network, respectively. The average throughput of a user $m$ in the hybrid network is then given by

$$
\begin{aligned}
\mathrm{E}\left[C_{m}\right]= & \mathrm{P}\left[m \in \mathcal{M}_{B}\right] C\left(S N R_{m i n}, B_{B}\right)+ \\
& \sum_{n \in \mathcal{N}} \mathrm{P}\left[m \in \mathcal{M}_{U}(n)\right] \mathrm{E}\left[C\left(\gamma_{e f f}(m, n), B_{m, n}\right)\right]
\end{aligned}
$$

where $\mathrm{P}\left[m \in \mathcal{M}_{B}\right]$ denotes the probability that user $m$ is in the coverage area of the broadcast network, $\mathrm{P}\left[m \in \mathcal{M}_{U}(n)\right]$ is the probability that user $m$ is connected to the $n$th station of the unicast network and $\mathcal{N}$ is the set of unicast stations outside the broadcast coverage area. Note that $\mathcal{N}$ depends on the size of the broadcast coverage area, i.e. on $S N R_{\text {min }}$. For the sake of simplicity the model is assumed to be symmetric and a uniform distribution of users is considered. Thus users are generated across area $[0,+R O I]$ (see Fig. 2). Let $X$ be the random variable characterizing the position of the users. The normalized probability density function (PDF) of $X$ then writes

$$
f_{X}(x)=\mathbf{1}_{[0,1]}(x)
$$

where $\mathbf{1}_{[0,1]}(x)$ is an indicator function of $X$. Then, the probabilities that user $m$ is connected to a broadcast and a unicast station are obtained as

$$
\begin{aligned}
\mathrm{P}\left[m \in \mathcal{M}_{B}\right] & =\mathrm{P}[X \in[0, R]] \\
\mathrm{P}\left[m \in \mathcal{M}_{U}(n)\right] & =\mathrm{P}\left[X \in\left[x_{n}-R_{U}, x_{n}+R_{U}\right]\right]
\end{aligned}
$$

where $x_{n}$ and $R_{U}$ are the normalized position and coverage radius of the $n$th station in the unicast network, respectively, and $R$ is the normalized broadcast coverage radius. Recall that $R$ depends on $S N R_{\text {min }}$ in (7). To calculate the distribution of the user's throughput for a station $n$ in the unicast network, the distribution of the distance $D$ and the SINR between a given station $n$ at a position $x_{n}$ and any user in the region of interest are successively derived. The distance $D$ between the position $x$ of a user and a station $n$ at position $x_{n}$ is given by $D_{n}=\left|x-x_{n}\right|$ which leads to the PDF of $D_{n}$,

$$
f_{D_{n}}(x)=\mathbf{1}_{\left[0, x_{n}\right]}(x)+\mathbf{1}_{\left[0,1-x_{n}\right]}(x)
$$

Substituting $d_{m, n}$ in (2) by $D_{n}$ and integrating according to (16) yields,

$$
f_{S I N R_{n}}(x)=\frac{\frac{\delta_{0}}{\alpha \gamma_{0}}\left(\frac{\gamma_{0}}{x}\right)^{1+\frac{1}{\alpha}} \times}{\left[\mathbf{1}_{\left[\phi\left(x_{n}\right),+\infty[\right.}(x)+\mathbf{1}_{\left[\phi\left(1-x_{n}\right),+\infty[\right.}(x)\right]}
$$

where $\gamma_{0}=P_{n, k} /\left(N_{0} \Delta_{f}+I_{k}\right), \delta_{0}=\lambda /(4 \pi)$ and $\phi(x)=$ $\gamma_{0}\left(\delta_{0} / x\right)^{\alpha}$. The distribution of the user's throughput for a station $n$ is then obtained by integrating (5) using (17)

$$
f_{C_{n}}(x)=\frac{A_{0} e^{x}\left(e^{x}-1\right)^{-\left(1+\frac{1}{\alpha}\right)} \times}{\left[\mathbf{1}_{\left[\psi\left(x_{n}\right),+\infty[\right.}(x)+\mathbf{1}_{\left[\psi\left(1-x_{n}\right),+\infty[\right.}(x)\right]}
$$

where $A_{0}=\delta_{0} \gamma_{0}^{\frac{1}{\alpha}} /(\alpha \beta), \psi(x)=\log \left(1+\gamma_{0}\left(\delta_{0} / x\right)^{\alpha}\right)$ and $\beta=B / \log (2)$ with $B$ the average bandwidth allocated per user by station $n$. The average throughput of a user $m$ connected to station $n$ is then given by

$$
\mathrm{E}\left[C\left(\gamma_{e f f}(m, n), B_{m, n}\right)\right]=\int_{x_{n}-R_{U}}^{x_{n}+R_{U}} x f_{C_{n}}(x) d x
$$

Finally the throughput maximization problem states

$$
\begin{aligned}
\max _{S N R_{\text {min }}} & M \times \mathrm{E}\left[C_{m}\right] \\
\text { s.t. } & \eta^{(s)} \geq \eta_{\text {min }}^{(s)} .
\end{aligned}
$$

$\mathrm{E}\left[C_{m}\right]$ is obtained from (12) using (6), (14), (15) and (19).

\section{Simulation}

The scenario and optimization problem presented in section III is considered here. Results are obtained according to the system parameters highlighted in the following section as reported in Table I. A minimum throughput $R_{s}=512 \mathrm{kbps}$ is required for a user to receive the service. 
TABLE I. SIMULATION SETTINGS

\begin{tabular}{|c|cc|}
\hline Parameter & \multicolumn{2}{|c|}{ Value } \\
\hline & Unicast $\quad$ Broadcast \\
\hline Network infrastructure & LPLT $\quad$ HPHT \\
Network layout & hexagonal grid $\quad$ single cell \\
Inter site distance & $1700 \mathrm{~m}$ & - \\
Resource allocation & round robin & - \\
Transmission power (EIRP) & $1.5 \mathrm{KW}$ & $10 \mathrm{KW}$ \\
Carrier frequency & $750 \mathrm{MHz} \quad 700 \mathrm{MHz}$ \\
Transmitter height & $40 \mathrm{~m} \quad 100 \mathrm{~m}$ \\
Receiver height & \multicolumn{2}{|c|}{$1.5 \mathrm{~m}$} \\
Propagation model & Hata model \\
Bandwidth & \multicolumn{2}{|c|}{$10 \mathrm{MHz}$} \\
Channel model & $3 \mathrm{GPP}$ typical urban \\
ROI size & \multicolumn{2}{|c|}{$30 \times 30 \mathrm{Km}^{2}$} \\
\hline Maximum number of users & \multicolumn{2}{|c|}{ uniform } \\
Users distribution & \multicolumn{2}{|c|}{$512 \mathrm{kbps}$} \\
\hline Service required throughput & \multicolumn{2}{|c|}{ linear contents } \\
Service type &
\end{tabular}

\section{A. Simulation settings}

A LTE unicast system is considered for the unicast network. The smallest radio resource unit that is allocated to a user is the LTE physical resources block (PRB) which is a group of 12 subcarriers of $15 \mathrm{KHz}$. The total bandwidth is divided in subchannels of $180 \mathrm{KHz}$, i.e. 1 PRB. The length of one PRB is $0.5 \mathrm{~ms}$ which is the length of a slot. A slot is composed of 7 OFDM symbols. The resource allocation is done in a time and frequency domain at each subframe. A subframe is composed of 2 slots. In this paper, a simple resource allocation strategy is assumed for the unicast network, namely round robin strategy.

For the broadcast network, we consider in this paper a dedicated LTE broadcast system, where all PRBs are assigned to all users covered by the broadcast network. This assumption is made to highlight the benefits of broadcast and unicast cooperation without any physical layer comparison. Further, this assumption allows to simulate the offloading cooperation scenario where the LTE broadcast mode (e.g. eMBMS) is improved and then embedded in DVB-T2 future extension frame as suggested in [3], [4].

The Vienna LTE-A downlink system level simulator [9] has been used to simulate the hybrid network presented in section II. Several features have been added to the initial version of the simulator to support the HPHT type of infrastructure. MonteCarlo simulations have been used to evaluate the metrics defined in section II.

\section{B. Simulation results}

Fig. 3 gives the service success rate as a function of the number of users. Results are presented for the unicast network, the broadcast network and the hybrid network with different sizes of the broadcast coverage area. Recall that the broadcast coverage radius is obtained from the $S N R_{\min }$ using (7).

First, observe in Fig. 3 that the unicast network, in a standalone mode, can not deliver popular services to all users. When the number of users increases, the amount of resource needed to garantee the targeted service throughput $R_{s}$ per user increases accordingly. Since the available resource remains limited, the unicast network can not guarantee the service to all users and the the service success rate degrades. For example

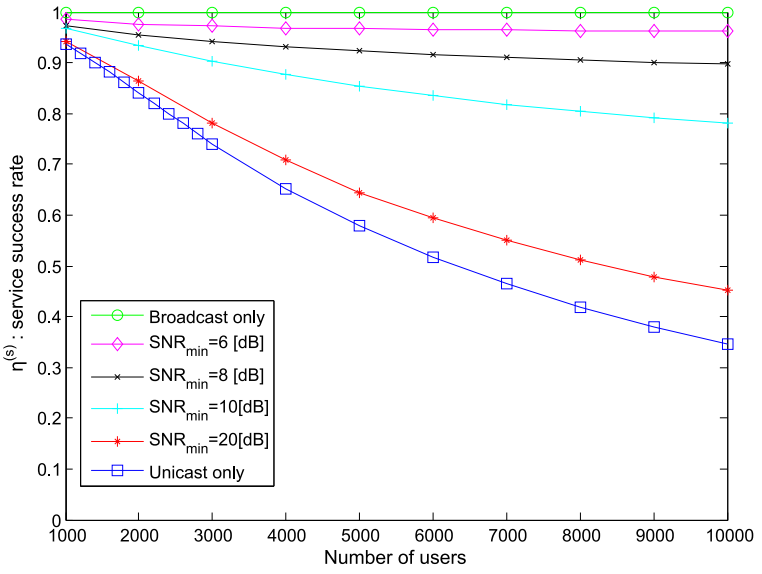

Fig. 3. Service success rate as a function of the number of users for the unicast network, the broadcast network and the hybrid network with different sizes of the broadcast coverage area.

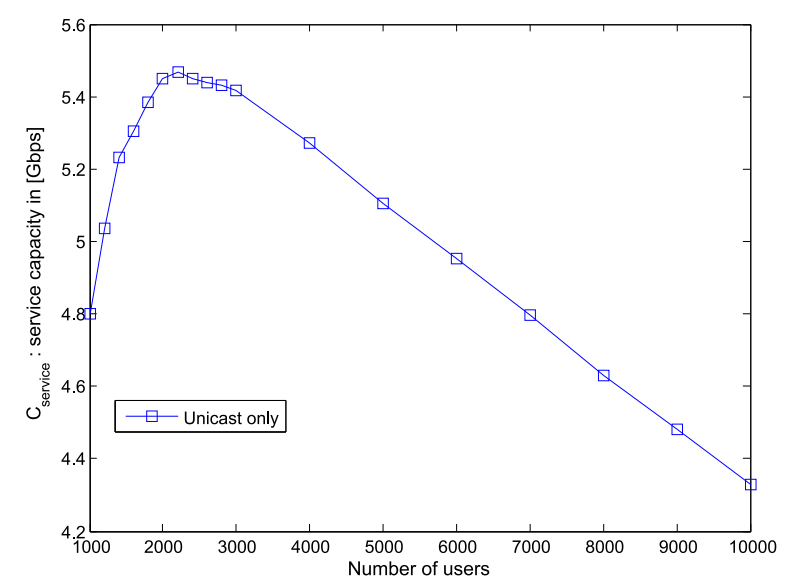

Fig. 4. Service capacity as a function of the number of users in the unicast network without cooperation with the broadcast network.

in Fig. 3 only $35 \%$ of the users receives the service for a total number of 10000 users in the network.

This degradation of the service success rate in the unicast network leads to the reduction of the service capacity of the network. This is shown in Fig. 4 which gives the evolution of the service capacity as a function of the number of users in the unicast network. Indeed, increasing the number of users in the unicast network decreases the service capacity. This is due to the strategy used for resource allocation. The round robin strategy, chosen for simulations, allocates the same amount of resource to all users. Consequently, when the number of users increases the throughput per user decreases because of the limited number of PRB.

On the other hand, it can be seen in Fig. 3 that broadcast and unicast cooperation improves the service success rate. Indeed, the cooperation gain depends on the size of the broadcast coverage area which is related to the targeted $S N R_{\text {min }}$ as we assume a constant transmit power. The service success rate achieved with the hybrid network when the number of users increases is better for a larger broadcast coverage area, i.e. for a lower $S N R_{\text {min }}$.

Furthermore, it turns out in Fig. 3 that the best service success rate is achieved when the entire region of interest is covered by the broadcast network, i.e. without cooperation 


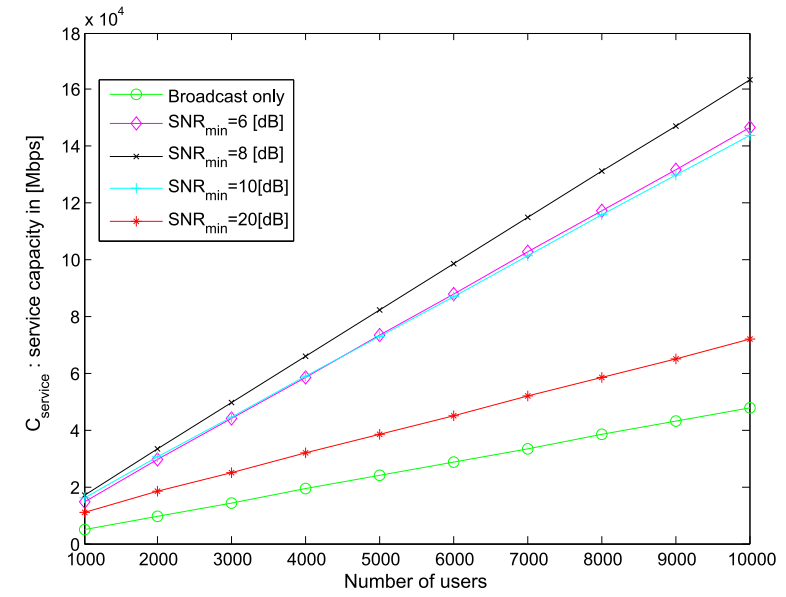

Fig. 5. Service capacity as a function of the number of users in the hybrid network for different sizes of the broadcast coverage area.

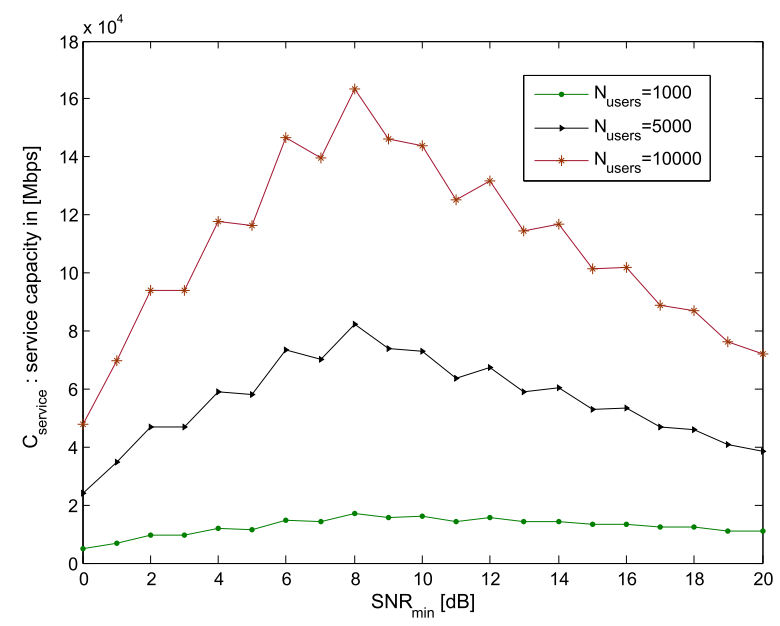

Fig. 6. Service capacity as a function of the size of the broadcast coverage area for different numbers of users in the hybrid network.

with the unicast network. However, to cover the entire region of interest, the broadcast network is planned for the worst case scenario, i.e. with a small $S N R_{\min }$. Recall that the throughput of a user connected to the broadcast network depends on the $S N R_{\text {min }}$. As a result, a low service capacity is achieved.

Fig. 5 shows the evolution of the service capacity as a function of the number of users for the broadcast network and for the hybrid network with different sizes of the broadcast coverage area. It can be seen that the lowest service capacity is achieved without cooperation. Moreover, it can be observed in Fig. 5 that reducing the broadcast coverage area increases the service capacity of the hybrid network only up to a maximum where the highest cooperation gain is achieved in term of throughput.

This latter observation is more relevant in Fig. 6 which depicts the service capacity as a function of the $S N R_{m i n}$, i.e. the size of the broadcast coverage area. Results are presented for different numbers of users in the hybrid network. Observe that the size of the broadcast coverage area that leads to the maximum service capacity does not depend on the number of users in the hybrid network. Here, the maximum service capacity is achieved with a $S N R_{\min }$ of $8 \mathrm{~dB}$.

Finally, these results suggest that a broadcast network is more appropriate than a unicast network to provide popular services. However, the entire region of interest need to be covered which leads to a low service capacity. On the other hand, broadcast and unicast cooperation maximizes the overall service capacity with a small degradation of the service success rate. This degradation is still acceptable since the maximum service capacity is achieved compared to a scenario without cooperation.

\section{CONCLUSION \& FUTURE WORKS}

In this paper, we investigated broadcast and unicast networks cooperation to provide linear services on mobile and portable devices from a planning point of view. We introduced an analytical approach to find an optimal operation mode, which maximizes the service capacity of the hybrid network taking into account the QoS requirements. Then through simulations, we showed the existence of an optimal operation point. Considering a cooperation scenario, this hybrid network model provides a great insight on the benefits of the cooperation. Thus, the mobile broadband data traffic generated by the highest requested services could be offloaded to the broadcast network while the broadcast network could avoid planning its network for the worst case scenario. The analytical approach proposed here can be used for planning of broadcast and unicast hybrid networks to find the best broadcast coverage radius with respect to the QoS requirements and the distribution of users. Our future works will be dedicated to further developments of the analytical approach introduced in this paper to find optimal values of other parameters of the networks.

\section{REFERENCES}

[1] C. V. N. I. (VNI), "Mobile data traffic forecast update 2013-2018," 2014. [Online]. Available: http://graphics8.nytimes.com/packages/pdf/ technology/cisco-mobile-forecast.pdf

[2] J. Monserrat, J. Calabuig, A. Fernandez-Aguilella, and D. GomezBarquero, "Joint Delivery of Unicast and E-MBMS Services in LTE Networks," IEEE Trans. Broadcast., vol. 58, no. 2, pp. 157-167, June 2012.

[3] M. Crussiere, C. Douillard, C. Gallard, M. Le Bot, B. Ros, A. Bouttier, and A. Untersee, "A Unified Broadcast Layer for Horizon 2020 Delivery of Multimedia Services," IEEE Trans. Broadcast., vol. 60, no. 2, pp. 193-207, June 2014.

[4] D. Rother, S. Ilsen, and F. Juretzek, "A Software Defined Radio based implementation of the Tower Overlay over LTE-A system," in Proc. IEEE BMSB, June 2014, pp. 1-6.

[5] A. Razzac, S. Elayoubi, T. Chahed, and B. El Hassan, "Planning of Mobile TV service in standalone and cooperative DVB-NGH and LTE networks," in 11th Int. Symp. WiOpt, May 2013, pp. 609-614.

[6] A. Razzac, S. Elayoubi, T. Chahed, and B. El-Hassan, "Comparison of LTE eMBMS and DVB-NGH mobile TV solutions from an energy consumption perspective," in IEEE 24th PIMRC, Sept 2013, pp. 16-20.

[7] A. Razzac, S. Elayoubi, T. Chahed, and B. El Hassan, "Impact of LTE and DVB-NGH cooperation on QoS of Mobile TV users," in Proc. IEEE ICC, June 2013, pp. 3672-3677.

[8] K. Brueninghaus, D. Astely, T. Salzer, S. Visuri, A. Alexiou, S. Karger, and G.-A. Seraji, "Link performance models for system level simulations of broadband radio access systems," in Proc. IEEE 16th PIMRC, vol. 4, Sept 2005, pp. 2306-2311 Vol. 4.

[9] J. C. Ikuno, M. Wrulich, and M. Rupp, "System Level Simulation of LTE Networks," in Proc. IEEE 71st VTC Spring, Taipei, Taiwan, May 2010, pp. 1-5. 